\title{
EPIDEMIOLOGY OF TYPHOID AND PARATYPHOID FEVER IN KATHMANDU : TWO YEARS STUDY AND TRENDS OF ANTIMICROBIAL RESISTANCE
}

\author{
Malla S*, Kansakar P*, Serichantalergs", Rahman $M^{* * *}$, Basnet $\mathrm{S}^{* * *}$ \\ * National Public Health Laboratory, ${ }^{* *}$ Armed Force Research Institute of Medical Sciences, Bangkok, ${ }^{* * *}$ ICDDR, Dhaka
}

\section{ABSTRACT}

Enteric fever is prevalent in developing countries including Nepal, where it still remains as a major health problem. Appropriate antibiotics are essential for the treatment of typhoid and paratyphoid fever. A prospective study was carried out to characterize the epidemiological features of enteric fever in Kathmandu, Nepal and to analyse the recent trend of antimicrobial resistance pattern of the Salmonella isolated from the cases of enteric fever from different hospitals in Kathmandu during June, 2002 to June, 2004. A total of 1469 Salmonella typhi and Salmonella paratyphi ' $A$ ' isolates collected during this period from five different hospital laboratories situated in Kathmandu were studied. The antimicrobial susceptibilities of the isolates towards Ampicillin (10 mcg), Chloramphenicol (30 mcg), Cotrimoxazole (25 mcg), Ciprofloxacin (5mcg) and Ceftriaxone (5mcg) were determined by standard disc diffusion technique and Agar dilution technique were used to determine the minimum inhibitory concentration (MIC) for Ampicillin, Ciprofloxacin, Chloramphenicol and Ceftriaxone. All the isolates tested were found to be sensitive to Ceftriaxone and Ciprofloxacin, the most commonly used antibiotic for treatment of enteric fever in Nepal. Of the total isolates studied, $15.5 \%$ from $2002,8 \%$ from 2003 and $3.45 \%$ from 2004 were found to be multidrug resistant (exhibiting resistance towards Ampicillin, Chloramphenicol and Cotrimoxazole). Of the total multi drug resistant Salmonella isolates, $92 \%$ were Salmonella typhi. All the multidrug resistant isolates were also further tested for susceptibilities towards Tetracycline (30 mcg), Nalidixic acid (30 mcg), Streptomycin (10units), Gentamycin (25mcg), Azithromycin $(15 \mathrm{mcg})$, Kanamycin $(30 \mathrm{mcg})$, Neomycin $(30 \mathrm{mcg}) .50 \%$ of the multi drug resistant Salmonella typhi were also resistant to Tetracycline. Plasmid analysis revealed that all of the Mutidrug resistant Salmonella typhi isolates with Tetracycline resistance harbored a large molecular weight $(147 \mathrm{~Kb})$ plasmid.

\section{Key Words: Enteric fever, Salmonella, Antibiotic, Plasmid, Multidrugresistant.}

\section{INTRODUCTION}

Enteric fever is prevalent in developing countries including Nepal, where it still remains as a major health problem. The annual global incidence of this disease has been estimated to be 21 million cases with more than 700.000 deaths. ${ }^{1}$ Areas with high incidence of typhoid include South East Asia, Africa and
Latin America. Countries like Indonesia, India and Nigeria report high mortality rate due to typhoid fever ranging from $12-32 \%$ in different studies. ${ }^{2,3,4}$ These countries seem to share several characteristics including rapid population growth, increased urbanization, inadequate human waste disposal, decreased water supply and overburdened health care system. ${ }^{5}$ Chloramphenicol was the drug of choice for enteric fever since its introduction in

Address for correspondence :

Ms. Palpasa Kansakar

National Public Health Laboratory, Teku, Kathmandu, Nepal

Email: nphl@wlink.com.np

Received Date : $12^{\text {th }}$ Jan., 2005

Accepted Date : $28^{\text {th }}$ June, 2005 
$1948 .{ }^{6}$ But in $1970 \mathrm{~s}$, plasmid mediated resistance to Chloramphenicol appeared and has been associated with outrbreaks in Latin America ${ }^{7}$ and Asia. ${ }^{8,9,10}$ With the emergence of chloramphenicol resistant strains, ampicillin and trimithoprim were considered suitable alternatives. ${ }^{11}$ Since 1989 , however, muti drug resistant (MDR) $S$ typhi strains that are no longer susceptible to these three first line antibiotics have emerged. ${ }^{12}$ In response to the emergence of muti-antibiotic resistant $S$. typhi , a number of studies have investigated the efficacies of newer compounds including expanded spectrum cephalosporins and fluoroquinolones. ${ }^{13,14}$ Ciprofloxacin is recommended as the drug of choice for typhoid fever since 1990s, but there are reports of $S$ typhi resistant to or with increased minimum inhibitory concentration (MIC) to ciprofloxacin from various parts including India, ${ }^{5}$ Korea, ${ }^{15}$ Vietnam. ${ }^{16}$

Antibiotic resistance in Salmonella typhi is often plasmid mediated. ${ }^{1}$ In particular, resistance to ampicillin, chloramphenicol, cotrimoxazole and tetracycline is often encoded by large molecular weight plasmid (about $180 \mathrm{~Kb}$ ) belonging to $\mathrm{H} 1$ incompatibility group. These plasmids are large $(\sim 180 \mathrm{~Kb})$ and conjugating and originate from Southeast Asia. ${ }^{1}$

This study aims at characterizing the recent epidemiological features of enteric fever in Kathmandu, Nepal and to analyze the recent trends of antimicrobial susceptibility of S. typhi and S. paratyphi A for the last two years.

\section{MATERIALS AND METHODS}

\section{Bacteria strains:}

Salmonella typhi and Salmonella paratyphi A strains isolated from cases of enteric fever at four major hospitals in KathmanduBir Hospital, Tribhuvan University Teaching Hospital (TUTH), Kanti Children's Hospital (KCH), and Maternity Hospital and the isolates from National Public Health Laboratory were included for the study. These strains included for analysis were isolated between June 2002 to July 2004. Bacterial identification were confirmed by biochemical testing on the following media: Kliger iron agar slants, Urea broth , Simmons citrate agar, Sulphide Indole Motility (SIM) medium (all from Oxoid) and serological agglutination with specific antisera (Denka Seiken, Japan). MDR Salmonella strains were defined as those strains possessing Chloramphenicol, Ampicillin and Cotrimoxazole resistance.

\section{Sensitivity Test:}

The antimicrobial susceptibility of the isolates towards Ampicillin (10 mcg), Chloramphenicol (30 mcg), Cotrimoxazole (25 mcg), Ciprofloxacin $(5 \mathrm{mcg})$ and Ceftriaxone (5 mcg) all from Oxoid, Hampshire were tested by Kirby Bauer's Disk diffusion technique. Agar dilution techniques were also used for determination of the minimum inhibitory concentration (MIC) for Ampicillin, Chloramphenicol, Ciprofloxacin and Ceftriaxone. All the multidrug resistant isolates were also further tested for susceptibilities towards Tetracycline $(30 \mathrm{mcg})$, Nalidixic acid (30mcg), Streptomycin (10units), Gentamicin (25mcg), Azithromycin (15 mcg), Kanamycin (30mcg) and Neomycin (30mcg).

\section{Plasmid study:}

Extraction of plasmid DNA was carried out for all the multidrug resistant Salmonella typhi by rapid alkaline lysis technique ${ }^{17}$ and the molecular weight of plasmid isolated was determined by agarose gel electrophoresis. ${ }^{18}$

\section{RESULTS}

\section{Epidemiologicalcharacteristics:}

A total of 1469 Salmonella (typhi and paratyphi A) isolates were collected during June 2002-June 2004 from four different hospitals and National Public Health Laboratory in Kathmandu. Distribution of the isolates collected is shown in Table I.

Table I : Distribution of total Salmonella isolates collected

\begin{tabular}{lccccc}
\hline Isolate & June-Dec, 2002 & Jan-June, 2003 & July-Dec, 2003 & Jan-June, 2004 & Total \\
\hline S. typhi & 106 & 427 & 319 & 187 & $1039(70.7 \%)$ \\
S paratyphi A & 23 & 161 & 141 & 105 & $430(29.3 \%)$ \\
Total Salmonella & 129 & 588 & 460 & 292 & 1469 \\
\hline
\end{tabular}

Table II : Age and Genderwise distribution of Salmonella analysed

\begin{tabular}{cccc}
\hline Age (yrs) & males & females & Total \\
\hline $0-9$ & $20 \%$ & $15 \%$ & $35 \%$ \\
$10-19$ & $20 \%$ & $11.8 \%$ & $31.8 \%$ \\
$20-29$ & $20 \%$ & $7 \%$ & $27 \%$ \\
$30-39$ & $3 \%$ & $1.6 \%$ & $4.6 \%$ \\
$40-49$ & $0.8 \%$ & $0.63 \%$ & $1.43 \%$ \\
$50-59$ & $0.3 \%$ & - & $0.3 \%$ \\
$>60$ & $0.1 \%$ & $0.22 \%$ & $0.23 \%$ \\
\hline Total & $\mathbf{6 4 \%}$ & $\mathbf{3 6 \%}$ & $\mathbf{1 0 0 \%}$ \\
\hline
\end{tabular}


The distribution of isolates by age, gender and seasons were also analysed. The infection occurred most frequently in the age group of 0-9 yrs followed by 10-19 and 20-29 years. Of the total Salmonella isolates analysed, $64 \%$ were from males and $36 \%$ were from females, indicating higher incidence of infection in males than in females (Table II).

The distribution of Salmonella isolates collected showed a clear seasonal clustering. The incidence rate was low in winter (from November to January), increased gradually from March and reached its peak in May-July (fig.1). However the number of isolates reported decreased from 2003 to 2004.

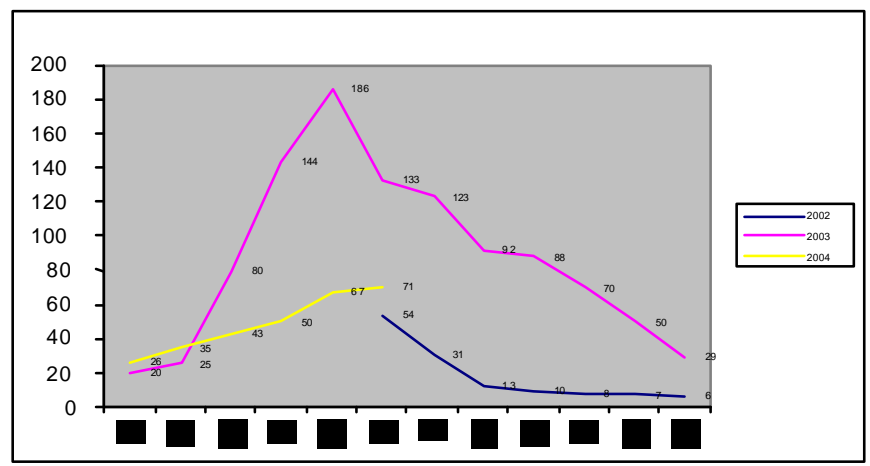

Fig. 1 : Monthly distribution of isolates analysed.

\section{Antimicrobial susceptibilities:}

All 1469 Salmonella typhi and paratyphi A included in the analysis were blood isolates. Of the total 1469 isolates, $70.7 \% \%$ were S. typhi and $29.3 \%$ were S. paratyphi A and $8.8 \%$ were multidrug resistant (MDR) strains, defined as resistant to ampicillin, chloramphenicol and cotrimoxazole. Resistance to Ciprofloxacin and Ceftriaxone were not seen. Of the total MDR Salmonella, 92\% were S. typhi. Distribution of multi-drug resistant strains in the past two years is shown in fig. 2 .

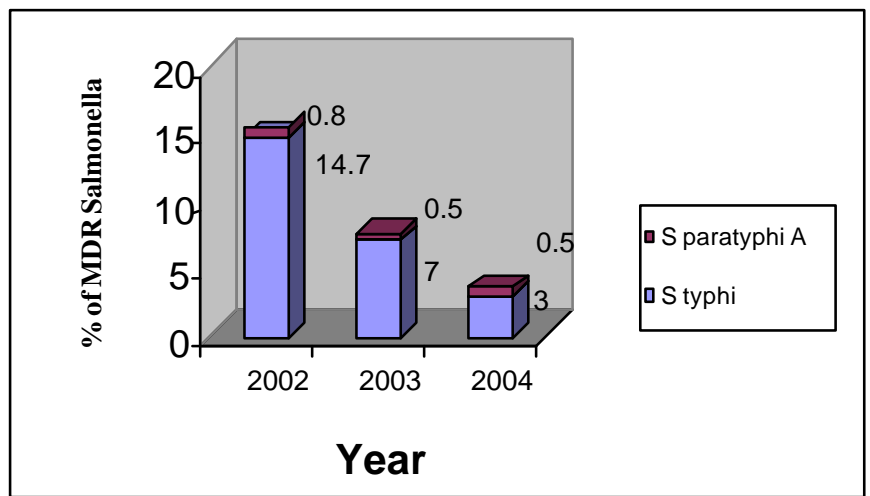

Fig. 2 : Percentage of Multi-drug resistant Salmonella typhi and paratyphi ' $A$ '.

Antimicrobial susceptibility test revealed that all the isolates tested were sensitive to Ciprofloxacin and Ceftriaxone. The overall resistance rate to ampicillin, chloramphenicol and cotrimoxazole were $32 \%, 30 \%$ and $30 \%$ in late half of 2002 , $32 \%, 13 \%$ and $15 \%$ in 2003 and $13 \%, 5 \%$ and $5 \%$ in the first half of 2004 respectively. (fig.3,4,5) For MDR strains, resistance

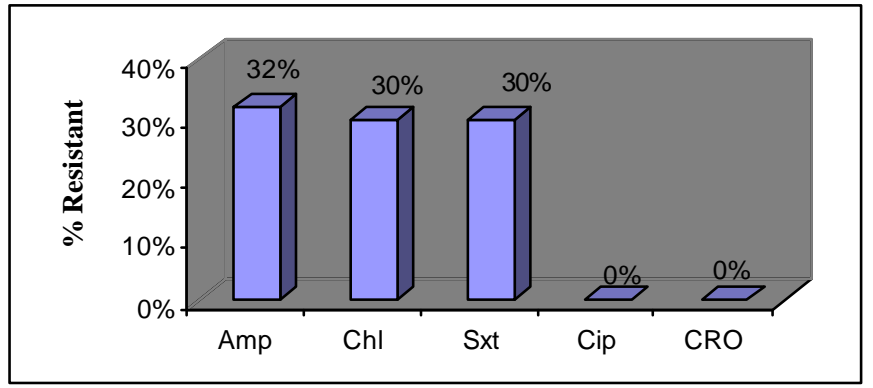

Fig. 3 : Antimicrobial susceptibility of Salmonella isolated in 2002

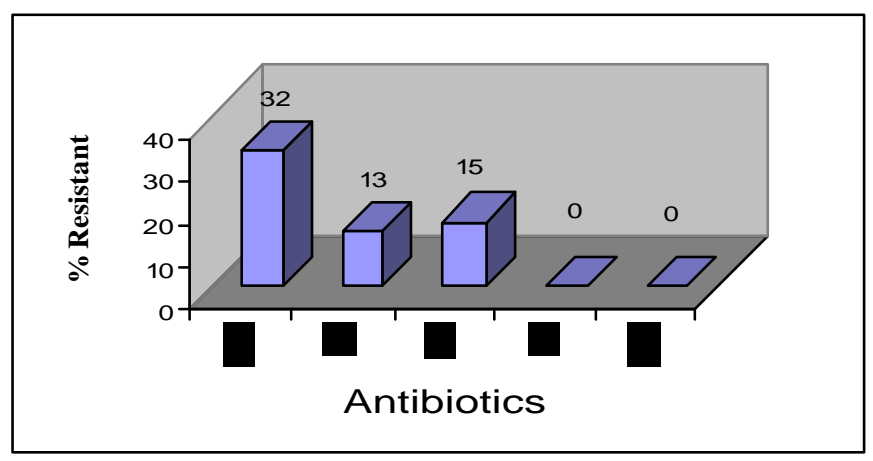

Fig. 4 : Antimicrobial susceptibility of Salmonella isolated in 2003

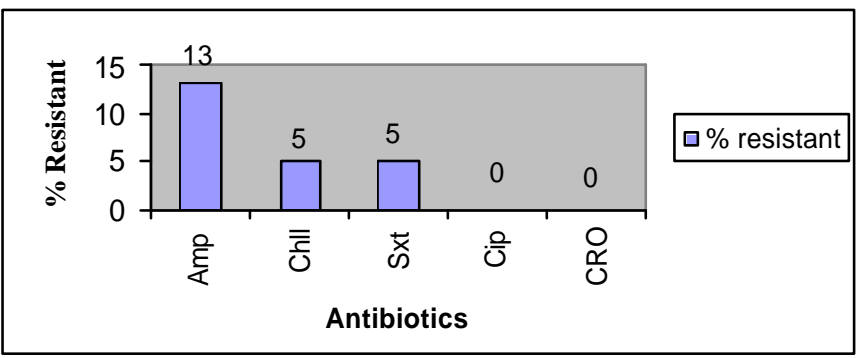

Fig. 5 : Antimicrobial susceptibility of Salmonella isolated in 2004

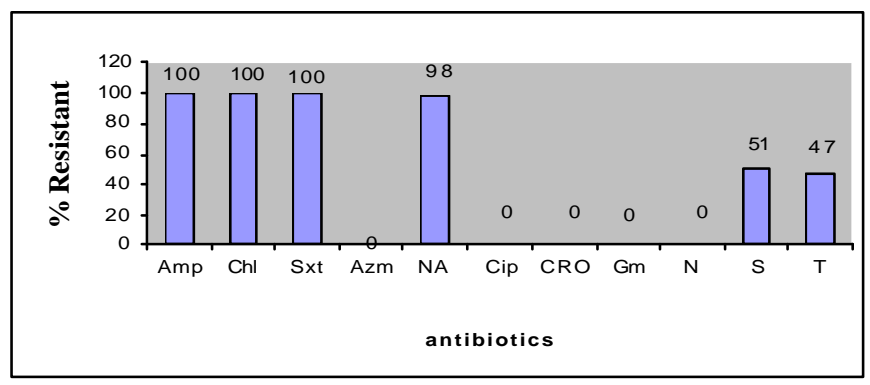

Fig. 6 : Antibiotic susceptibility pattern of multi drug resistant Salmonella

rate to Azithromycin, Nalidixic acid, Gentamicin, Neomycin, Streptomycin, Tetracycline and Kanamycin were 0\%, 98\%, 0\%, $0 \%, 51 \%, 50 \%$ and $0 \%$ respectively. (fig. 6)

\section{Plasmid size:}

All the MDR Salmonella typhi strains were further processed for plasmid DNA extraction and analysed by agarose gel electrophoresis for the presence of large molecular weight plasmid. It was found that all the MDR S. typhi strains with 
typhi in Asia for the past decade. But acquired drug resistance to this drug has posed a serious problem for the treatment of enteric fever. Single point mutation in the quinolone resistancedetermining region (QRDR) of the topoisomerase gene Gyr A in Salmonella usually leads simultaneously to resistance against Nalidixic acid and decreased Ciprofloxacin susceptibility (5).However, in our study all the isolates were sensitive to Ciprofloxacin (MIC $<1 \mathrm{mcg} / \mathrm{ml})$ although Nalidixic acid resistance among MDR Salmonella was very high (98\%).

Multi-drug resistance was known to be mediated by a large sized conjugable plasmids and several reports suggested the international transfer of R-plasmids. Reports suggest that these plasmids, which belong to the Inc HI incompatibility group, frequently encode resistance to Chloramphenicol, tetracycline resistance harbored a large molecular weight plasmid of $147 \mathrm{~Kb}$. (Fig.7).

\section{DISCUSSION}

This study provides comprehensive information on the epidemiology of enteric fever in Kathmandu, Nepal and the recent trends in antimicrobial resistance of the causative agents. The study shows that majority of enteric fever in Kathmandu is caused by $\mathrm{S}$ typhi (70.7\%), follwed by S paratyphi-A (29.3\%). While no cases of enteric fever caused due to S. paratyphi-B and/or S. paratyphi-C was reported from any of the hospitals/ laboratory included in the study. The infection was prevalent in the age group of $0-9$ yrs age followed by $10-19$ yrs and the incidence was higher in the male population (64\%) in comparison to female population (36\%). The number of isolates reported each month showed that enteric fever in Kathmandu occurred sporadically throughout the year with marked peaks in the summer and rainy seasons (May-July).

The increase in multi-drug resistant Salmonella is a huge problem in developing countries. The incidence rates of MDR strains were $26 \%$ in UK and $17 \%$ in USA, but infections have been in patients with a recent history of travel to India or Pakistan. ${ }^{19,20}$ In India $64.5 \%$ of S typhi was reported as being MDR in 1993. ${ }^{21}$ MDR S. typhi strains were first reported in Nepal in 2002 during Bharatpur outbreak of enteric fever in Chitwan, Nepal in May-June, 2002. This outbreak of enteric fever in Bharatpur, 2002 was reported to be a large single source outbreak due to multidrug resistant $\mathrm{S}$. typhi. ${ }^{22}$ Since then the necessity of monitoring the antimicrobial resistance in Salmonella initiated this study. In this study, the overall percentage of multi-drug resistant strains was $8.8 \%$, however the yearly breakdown of the MDR strains showed a gradual decrease, 2002 (15.5\%), 2003 (7.5\%) and 2004 (3.5\%). Ciprofloxacin is the drug of choice for multi drug resistant $\mathrm{S}$. study on MDR S typhi strains in Vellore, India has reported plasmids belonging to IncHI group, specifically IncHI1 and calculated as being between 140 and $170 \mathrm{~Kb} .{ }^{1}$ The plasmids detailed in the current study were also estimated as being 147 $\mathrm{Kb}$. Although, conjugational study for transfer of drug resistance and pulsed field gel electrophoresis (PFGE) based analysis couldnot be carried out in this investigation, recent studies indicate multi drug resistant S. typhi with plasmid mediated block resistance to chloramphenicol, ampicillin and cotrimoxazole. However, further genotypic studies are needed to analyze the spread of MDR strains in Kathmandu.

\section{CONCLUSION}

In conclusion, we have noticed MDR Salmonella isolated from cases of enteric fever in Kathmandu showing resistance to all three first line antibiotics used for treatment-Ampicillin, Chloramphenicol and Cotrimoxazole. Although there are reports of gradual increase in the incidence of resistance to newer drugs like quinolones, ${ }^{5,15,16,26,27}$ all the isolates tested in our study are still sensitive to Ciprofloxacin (MIC value $<=1 \mathrm{mcg} / \mathrm{ml}$ ) and Ceftriaxone (MIC value $<=8 \mathrm{mcg} / \mathrm{ml}$ ). However, there are reports that when the sensitivity pattern indicates resistance to nalidixic acid which is a marker for delayed clinical response to fluoroquinolones, it is necessary to increase the oral dose of ciprofloxacin or treat the patient with third generation cephalosporin like Ceftriaxone. ${ }^{28,29}$ In the present study, all the S. typhi strains were sensitive to ciprofloxacin and Ceftriaxone. Since strains resistant to Ciprofloxacin and Ceftriaxone werenot identified, surveillance for resistance to first line antibiotics and fluoroquinolones should be continued.

\section{ACKNOWLEDGEMENT}

This study was supported by a grant of USAID/ Nepal mission. 


\section{REFERENCES}

1. Philippa M. A. Shanahan, Mary V. Jesudason,Christpher J. Thomson,Sebastian G. B. Amyes,Molecular Analysis of and Identification of Antibiotic Resistance Genes in Clinical Isolates of Salmonella typhi in India, Jour of Clinical Microbiology,June 1998;36-6.

2. Edelman R, Levine MM, Summery of an International workshop on Typhoid fever, Rev Infect Dis, 1986, May-Jun:8(3): 329-40.

3. Anderson ES, Joseph SW, Nasution Q. Febrile illness resulting in hospital admission, A bacteriological \& serological study in Jakarta Indonesia Am. J Trop .Med. Hyg. 1976; 25:116-21.

4. Gulati PD, Saxena SN, Gupta PS et al Changing pattern of typhoid fever Am.J.Med 1968; 45:544-8.

5. Lathi N, Sudarshana J, Changing sensitivity pattern of Salmonella tyhpi in calicut,Calicut Medical Journal; 2004; 2(1):e2.

6. Islam A, Butler T, Kabir I, Alam N H, Treatment of typhoid fever with ceftriaxone for 5 days or chloramphenicol for 14 days: a randomized clinical trial. Antimicrob. Agents Chemother. 37:1572-1575.

7. Olark J, Galindo ES. Styphi resistant to C, A and other antimicrobial agents: strains isolated in extensive typhoid fever epidemic in Mexico. Antimicrob. agents chemother. 1973; 4: 597 - 601.

8. Panicker CK, Vimala KN Transferable Chloramphenicol resistance in Salmonella. Nature. 1972; 239: 109 - 10.

9. Lanpe PM Mansuwan P Duangman C. Chloramphenicol resistant typhoid. Lancet 1974; 1: $623-4$.

10. Sudarsana J, Lathi N, Devi K I. Multidrug resistant Salmonella typhi in Calicut South India. I JMR ,95, March 1992, 68 - 70.

11. Du Pont, H.L., Quinolones in Salmonella typhi infections; Drugs, 1993,45:119-124.

12. Jesudasan MV, Malathy B John TJ. Trend of increasing levels of MIC of Ciprofloxacin to S. typhi. IJMR, 1996 May, 103: 247 - 49.

13. Gulati S., R K Marwaha, S Singhi, A. Ayyagari, L Kumar, Third generation cephalosporins in multi-drug resistant typhoid fever, Indian Pediatr, 1992, 29: 513-516.

14. Mathai D., G.C. Kudwa, J.S. Keystone, P.E. Kozarsky, M.V. Jesudason, M. K. Lalitha, A Kaur, M Thomas, J John, B M Pulimood, Short course of ciprofloxacin in enteric fever, J Assoc Physicians India ,1993, 41: 74287430.

15. Sunmi Y., Hyunjoo P., Jeong B., Youn H K., Shukho K., Bok K. L., Epidemiology of Salmonella enterica serotype Typhi Infections in Korea for Recent 9 Years: Trends of Antimicrobial Resistance, J Korean Med Sci; 2004,19:15-20.
16. Phung L. V, H Ryo, Nomura T, Specific gyr A mutation at codon 83 in Nalidixic acid resistant salmonella enterica serovar typhi strain isolated from Vietnamese patients, Letter to the editor, Antimicrobial agents and Chemotherapy, June 2002, Vol 46 No 22062 - 63.

17. Birnboim H.C. and Doly J., A rapid alkaline extraction procedure for screening recombinant plasmid DNA, Nucleic Acids Research, 1979, Vol 7, Number 6.

18. Wilson K., Walker J, Electrophoretic techniques, Practical Biochemistry: Principles and Techniques, $5^{\text {h }}$ edt., 2000, 607-610.

19. Ackers M L, Puhr N D, Tauxe R V, Mintz E D. Laboratory based surveillance of Salmonella serotype typhi infections in the United States: antimicrobial resistance on the rise. JAMA 2000; 283: 2668-73.

20. Threlfall E J, Ward L R,. Decreased susceptibility to ciprofloxacin in Salmonella enterica serotype Typhi, United Kingdom. Emerg Infect Dis 2001; 7: 448-50.

21. Pillai P K, Prakash K. current status of drug resistance and phage types of Salmonella typhi in India. Indian J Med Res 1993; 97: 154-8.

22. Lewis M., Mason C, Pitarangsi C, Chuanak N, Pandey P, Chaudhary B $N$, Laskar R, Patowary A, Shrestha C D, Malla S.A large single source outbreak of multi-drug resistant typhoid fever in Bharatpur, Chitwan, Nepal, 2003, Souvenir-Book of Abstracts, Fourth Congress of assoc. of clin. Pathologists of Nepal, feb 21-22, 2003.

23. Finch, M. J., A. franco, E Gotuzzo, C. Carillo, L. Benavente, S.S. Wasserman, M.M. Levine, J G Morris, Jr., Plasmids in salmonella tyhpi in Lima, Peru, 1987-1988 : epidemiology and lack of association with severity of illness or clinical complications. Am J. Trop. Med. Hyg., 1992, 47: 390-396.

24. Goldstein, F.W., J.C. Chumpitaz, J.M. Guevera, Plasmid mediated resistance to multiple antibiotics in S typhi. J. Infect. Dis, 1986, 153(2): 261:266.

25. Karmaker, S., D. Biswas, N M Shaikh, S. K. Chatterjee, V.K. Kataria, R Kumar. Role of a large plasmid of Salmonella typhi encoding multiple drug resistance. J. Med. Microbio, 1991, 34: 149-151.

26. Mirza, S.H., C.A. Hart. Plasmid encoded multiple drug resistance in Salmonella typhi from Pakistan, Ann. Trop. Med. Parasitol, 1993. 87:373377.

27. Deodhar L, Bhave S, Agarwal A. 'Bacteriophage typing of multi drug resistant Salmonella typhi in paediatric patients'. Bombay Hospital Journal 1993; 35 (4): 14-15.

28. Rodrigues C, Mehta A, Joshi VR. Quinolone resistant enteric feverproblems and remedies. JAPI 1998; 46 (8): 751-52.

29. Miller SI, Hohmann E, Pagues DA. Chapter 200 on Salmonella (including Salmonella typhi) in 'Infectious Diseases' Editors : GL Mandell, JE Bennett, R Dolin, Churchill Livingstone, 4th Edition. 1995; 2013-33.

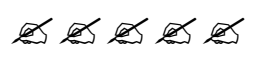

